\title{
Intravitreal Aflibercept Treatment Strategies in Routine Clinical Practice of Neovascular Age- Related Macular Degeneration in Belgium: A Retrospective Observational Study
}

Joke Ruys · Els Mangelschots · Julie Jacob · Filip Mergaerts •

Alexandra Kozyreff · Werner Dirven

Received: July 22, 2020 / Accepted: September 3, 2020 / Published online: September 18, 2020

(C) The Author(s) 2020

\section{ABSTRACT}

Introduction: STELLAR was a Belgian, multicentre, retrospective, observational chart review that described the utilization (number of injections and treatment regimen) and effectiveness of intravitreal aflibercept (IVT-AFL) in patients

Digital Features To view digital features for this article go to https://doi.org/10.6084/m9.figshare.12906812.

Electronic supplementary material The online version of this article (https://doi.org/10.1007/s40123020-00300-7) contains supplementary material, which is available to authorized users.

J. Ruys $(\square)$

Department of Ophthalmology, ZNA Middelheim, Antwerp, Belgium

e-mail: joke.ruys@zna.be

E. Mangelschots

Oogartsenpraktijk Alken, Alken, Belgium

J. Jacob

Department of Ophthalmology, UZ Leuven, Leuven, Belgium

F. Mergaerts

Oogartsenpraktijk Scherpenheuvel, Aarschot, Belgium

A. Kozyreff

Department of Ophthalmology, Cliniques

Universitaires de Saint-Luc, Brussels, Belgium

W. Dirven

Department of Ophthalmology, AZ Turnhout, Turnhout, Belgium with anti-vascular endothelial growth factor (VEGF) treatment-naïve neovascular age-related macular degeneration (nAMD) during the first 12 months of IVT-AFL treatment.

Methods: Patients initiating IVT-AFL between July 2013 and July 2017 were included in STELLAR. Primary endpoints were number of visits and IVT-AFL injections, and number of patients who received $\geq 7$ versus $<7$ IVT-AFL injections during the first 12 months of treatment.

Results: A total of 337 patients completed $\geq$ 12 months of IVT-AFL treatment. The mean number of visits and mean number of injections during the first 12 months was 9.8 and 7.1 injections, respectively (64\% received $\geq 7$ injections). Overall, $96 \%$ of patients received $\geq 3$ initial monthly injections. Of the 337 patients, 180 received VTAFL as needed (pro re nata), 141 received it as treatand-extend dosing and 16 received it as fixed dosing. The proportion of patients who received treatand-extend dosing increased year-on-year. Mean best-corrected visual acuity (BCVA) ( \pm standard deviation) was $61.6( \pm 14.9)$ Early Treatment Diabetic Retinopathy Study (ETDRS) letters at baseline and improved by +3.9 and +5.7 ETDRS letters at 3 and 12 months, respectively. Mean BCVA improvement was numerically greater in patients who received $\geq 7$ versus $<7$ injections during the first 12 months 7 (+ 6.5 vs. +4.4 ETDRS letters) and in patients who received $\geq 3$ versus $<3$ initial monthly injections (+ 5.2 vs. -0.25 ETDRS letters [3 at months]; +5.9 vs +1.2 ETDRS letters [at 12 months]). No specific adverse events were reported. 
Conclusion: Most patients in this Belgian study received $\geq 7$ IVT-AFL injections during a mean of 9.8 visits over the 12 months assessed. IVTAFL was an effective treatment for nAMD in clinical practice, with numerically higher BCVA gains in patients receiving $\geq 7$ versus $<7$ injections over the first 12 months and $\geq 3$ versus $<3$ injections in the first 3 months.

Keywords: Aflibercept; Age-related macular degeneration; Intravitreal injections; Retina; Retina-Medical therapies

\section{Key Summary Points}

\section{Why carry out this study?}

Different treatment patterns have been applied using anti-vascular endothelial growth factor agents for the treatment of neovascular age-related macular degeneration (nAMD), such as fixed, as needed and treat-and-extend treatments. Previous studies have shown that proactive regular treatment in the first year is associated with better best-corrected visual acuity (BCVA) outcomes.

The aim of our study was to describe (the evolution in) treatment strategies used in routine clinical practice in Belgium during the first 12 months of intravitreal aflibercept (IVT-AFL) use in treatment-naive nAMD patients over the period 1 July 2013 to 1 July 2017.

\section{What was learnt from the study?}

Patients received a mean of 7.1 injections of IVT-AFL during the first 12 months of treatment; $96 \%$ of patients received $\geq 3$ initiation injections and $64 \%$ received $\geq 7$ injections. Mean change in BCVA (ETDRS letters) from baseline was +3.9 (at 3 months) and +5.7 (at 12 months), which is comparable to other real-world-evidence studies with IVT-AFL. There were numerically higher BCVA gains in patients receiving $\geq 7$ injections over the first 12 months and $\geq 3$ injections in the first 3 months.
There was a shift in the usage pattern in the 4 years of the study from a reactive (2013) to a proactive treatment (2017) with good functional outcomes.

\section{DIGITAL FEATURES}

This article is published with digital features to facilitate understanding of the article. To view the digital features for this article go to https:// doi.org/10.6084/m9.figshare.12906812.

\section{INTRODUCTION}

Neovascular age-related macular degeneration (nAMD) accounts for $>90 \%$ of all cases of blindness attributable to age-related macular degeneration $[1,2]$. Treatment with intravitreally injected drugs, such as intravitreal aflibercept (IVT-AFL) and ranibizumab, which target vascular endothelial growth factor (VEGF), has been established in international guidelines as the first-line treatment of nAMD of preference based on improvements in visual and anatomic outcomes [3-5]. Prior to the availability of IVTAFL, anti-VEGF agents were routinely given in a reactive manner as needed (pro re nata $[\mathrm{PRN}]$ ), with patients receiving anti-VEGF treatment in response to signs of disease reactivation. However, the administration of intravitreal ranibizumab PRN has been shown to result in lower mean gain in visual acuity compared with fixed monthly regimens (proactive treatment) in clinical trial or real-world clinical practice settings $[6,7]$.

The efficacy of IVT-AFL in the treatment of nAMD was first demonstrated in two phase 3 randomized, active-controlled trials in patients with nAMD (VIEW1 and VIEW2). In these studies, IVT-AFL given in a proactive fixed dosing regimen (initially as three monthly injections then every 8 weeks thereafter) demonstrated improvements in visual acuity over the first year that were comparable with monthly ranibizumab [8]. During weeks 52 through 96, patients received their original dosing assignment using a PRN regimen with 
defined retreatment criteria and mandatory dosing at least every 12 weeks. At 96 weeks, IVTAFL every 8 weeks achieved visual acuity outcomes similar to those of ranibizumab, but with an average of five fewer injections than ranibizumab [9]. In 2018, based on the results of the ALTAIR study, the IVT-AFL dosing recommendations were expanded to allow proactive treatand-extend (T\&E) dosing in year 1, after three initial monthly injections and then an injection 8 weeks later $[10,11]$.

The effectiveness and safety of IVT-AFL in clinical practice has been shown in observational studies across Europe, including Germany, France and the UK [12-16]. Real-world clinical practice studies in France (RAINBOW) and Germany (PERSEUS) showed that proactive regular treatment in the first year (defined as $\geq 7$ injections in RAINBOW and PERSEUS) given every 4 weeks $(-1 /+2$ weeks) for the initial three injections, followed by injections every 8 weeks $(-2 /+4$ weeks in PERSEUS and $-3 /+4$ weeks in RAINBOW) was associated with better visual acuity outcomes compared with not receiving regular treatment $[14,16]$. Similar findings have been shown in studies carried out in the UK $[12,13,15]$.

Currently, structured real-world clinical practice data regarding the use of IVT-AFL in nAMD in Belgium following its initial approval in 2013 are lacking. The objective of STELLAR, a 4-year observational study, was to describe treatment strategies used in routine clinical practice in Belgium during the first 12 months of IVT-AFL treatment in treatment-naive patients diagnosed with nAMD who started IVT-AFL treatment between 1 July 2013 and 1 July 2017 and to describe any evolution of those practices over that period.

\section{METHODS}

\section{Study Design}

STELLAR was a multicentre, observational, retrospective chart review of data from eight centres geographically spread across Belgium that involved treatment-naïve patients diagnosed with nAMD who initiated reimbursed IVT-AFL treatment between 1 July 2013 and 1 July 2017 . IVT-AFL was administered according to the physician's routine clinical practice which, according to the prescribing recommendations, should include three initial monthly injections irrespective of the subsequent treatment regimen used.

Data were collected between 9 November 2018 and 26 March 2019 in an aggregated manner per centre. Approvals from independent ethics committees were obtained for all participating centres prior to study start (Electronic Supplementary Material Table). In order to evaluate the evolution in usage patterns and dosing regimen, the population of patients who completed at least 12 months of treatment were subdivided for certain endpoints into four subgroups, based upon the start date of IVT-AFL treatment.

\section{Patients}

Patients were men and women aged $\geq 18$ years (no upper age limit) diagnosed with nAMD who were anti-VEGF treatment naïve and who started IVT-AFL treatment between 1 July 2013 and 1 July 2017. Patients were excluded if they were participating in an ophthalmological investigational programme/clinical trial with interventions outside of routine clinical practice during the treatment period.

\section{Endpoints}

The primary endpoints were the number of IVTAFL injections and visits as well as the number of patients who received $\geq 7$ versus $<7$ IVT-AFL injections during the first 12 months of treatment, both calculated for patients who completed at least 12 months of IVT-AFL treatment.

For patients who completed at least 12 months of IVT-AFL treatment, secondary endpoints were the number of IVT-AFL injections administered during the first 3 months of treatment ( -1 week/ 2 weeks, equating to 83-104 days); number of patients starting treatment in each study year; dosing regimen (PRN, T\&E or fixed, as identified by investigators using their clinical discretion and based on 
the information in the patient dossier) during the first 12 months of treatment ( -1 week/ +3 weeks); mean baseline best-corrected visual acuity (BCVA measured using Early Treatment Diabetic Retinopathy Study [ETDRS] letters); and change in visual acuity after the first three initial doses and first 12 months of treatment.

Secondary endpoints (calculated based on all enrolled patients) also included the number of patients treated for both eyes, the number of patients who discontinued IVT-AFL treatment during the first 12 months and reasons for discontinuation, where applicable. Additional analyses will be performed for the subgroups 2013-2014, 2014-2015, 2015-2016 and 2016-2017 based on the start date of IVT-AFL treatment (year 1, year 2, year 3, and year 4, respectively). All described endpoints were based on the investigator's clinical discretion using the information in the patient dossier. For each patient, only the eye that was treated initially was considered.

Healthcare professionals reported adverse events (AEs) encountered through review of their patients' charts. According to clinical practice guidelines in Europe for observational studies based on secondary use of data, individual reporting of adverse reactions is not required [17].

\section{Data Analysis}

A sample size of approximately 400 patients who completed the first 12 months of treatment was estimated to be sufficient to ensure statistical power to analyse the primary objective. Therefore, approximately 550 patients were planned for enrolment to ensure that 400 patients completed 1 year of treatment.

Data were collected by investigators retrospectively from medical records. Aggregated data, per site and for certain variables by subgroups, were generated for each endpoint and provided to the study sponsor, which had no access to the individual-level patient data. Site data were combined to calculate outcomes for the overall population and subgroups. For the continuous variable, change from baseline in visual acuity, weighted means, weighted standard deviation (SD) and 95\% confidence intervals (CI) were calculated for the overall population and for subgroups. Weighted descriptive statistics were used to account for the difference in sample sizes across the different sites.

Due to the observational and retrospective nature of this study, no formal hypothesis testing was performed, and thus all statistical analyses should be considered exploratory in nature.

\section{RESULTS}

\section{Patients}

A total of 411 patients from eight centres across Belgium were enrolled in the study, of whom 337 patients continued treatment for $\geq 12$ months and were included in further analyses. Although the sample size is smaller than initially planned, it is still sufficient from a statistical point of view to answer the primary research question. Fifty-six patients initiated treatment in year 1 (July 2013-June 2014), 109 in year 2 (July 2014-June 2015), 111 in year 3 (July 2015-June 2016) and 61 in year 4 (July 2016-June 2017). Overall mean \pm SD baseline BCVA was $61.6 \pm 14.9$ ETDRS letters $(95 \% \mathrm{CI}$ 60.0-63.2). Seventy-four patients discontinued IVT-AFL treatment during the first 12 months of treatment (Fig. 1), with the main reasons for discontinuation within the first year being inactive choroidal neovascularization $(n=21$; $28 \%)$ or a lack of efficacy $(n=19 ; 26 \%)$.

\section{Treatment Strategies}

The mean $( \pm S D$ for all variables presented here) number of injections in the total population during the first year was $7.1 \pm 2.3$ (95\% CI $6.8,7.3)$, given during a mean of $9.8 \pm 2.1(95 \%$ CI 9.6-10.0) treatment visits; the mean number of injections during the first 3 months was $3.1 \pm 0.4$ (95\% CI 3.0-3.1). Overall, of the 337 patients, $64 \%$ (216) received $\geq 7$ injections during the first 12 months (Fig. 2 ) and 96\% ( $n=$ 325) received $\geq 3$ injections during the first 


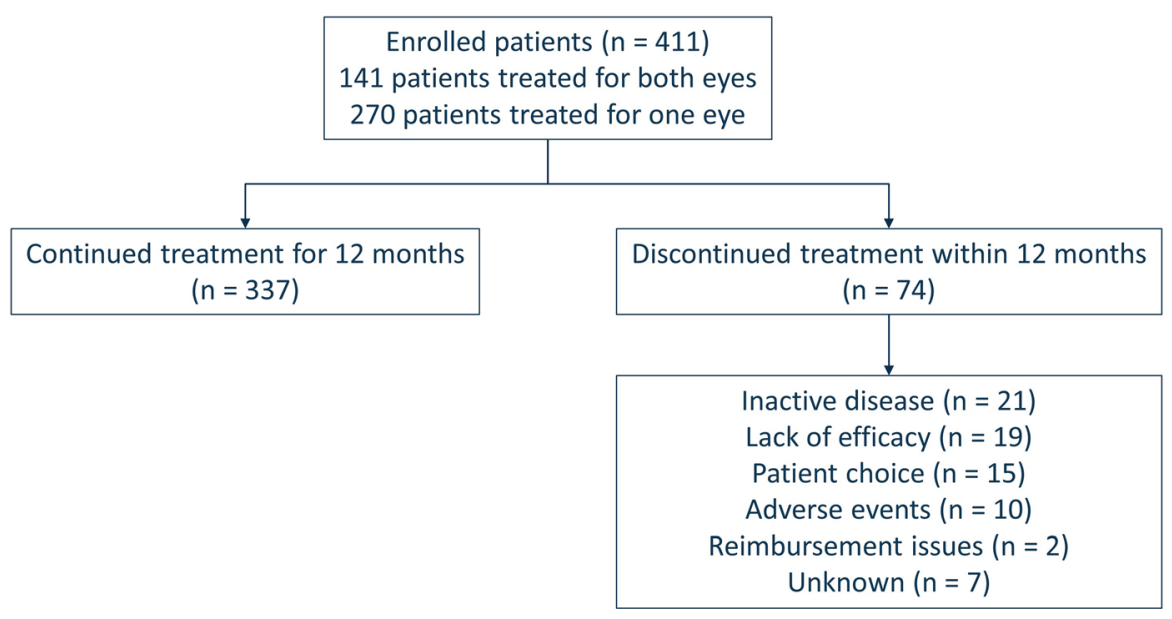

Fig. 1 Flow diagram of patient disposition in the study

3 months (only 12 patients received $<3$ injections); $63 \%$ of patients $(n=212)$ received both $\geq 3$ injections during the first 3 months and $\geq 7$ injections during the first year.

During the observation period, the proportion of patients with $\geq 7$ injections increased over time from $48 \%$ of patients $(27 / 56)$ initiating treatment in year 1 to $67 \%$ of patients (41/ 61) initiating treatment in year 4 . In the first 12 months of treatment, $53 \%$ of patients (180/ $337)$ were treated with a PRN dosing regimen, $42 \%(141 / 337)$ with a T\&E regimen and 5\% (16/ 337) with a fixed dosing regimen. There was a change away from a PRN dosing regimen toward a T\&E dosing regimen over the duration of the study, with $62 \%$ of patients who started their treatment in year 4 being treated by $T \& E$,

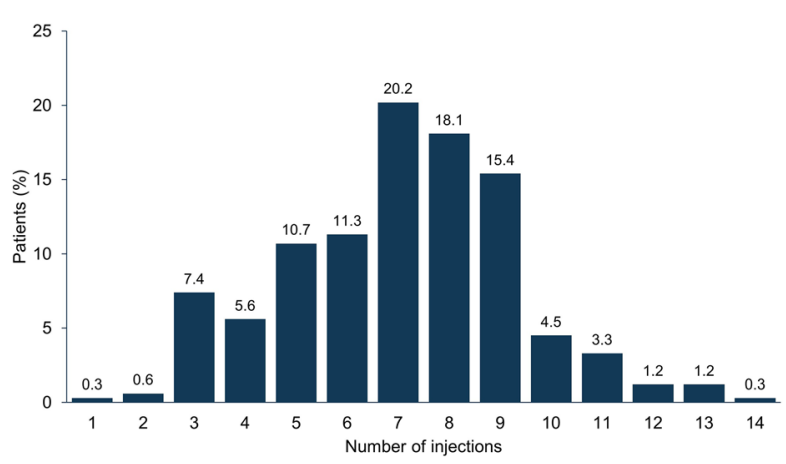

Fig. 2 Distribution of number of intravitreal aflibercept injections over first 12 months of treatment compared with $16 \%$ of patients initiating treatment in year 1 (Fig. 3).

\section{Functional Outcomes}

From an overall mean $( \pm$ SD for all variables presented here) baseline BCVA of $61.6 \pm 14.9$ ETDRS letters, mean \pm change in BCVA was $3.9 \pm 13.7$ letters (95\% CI 2.4-5.3) after three initial doses and $5.7 \pm 15.0$ letters (95\% CI 4.1-7.3) after 12 months (Fig. 4). Mean baseline BCVA was similar to the overall population in patients who received $<7$ or $\geq 7$ injections within the first 12 months. In 216 patients who received $\geq 7$ injections in the first 12 months, mean BCVA improved by $6.5 \pm 15.0$ ETDRS letters (95\% CI 4.5-8.5) after 12 months versus

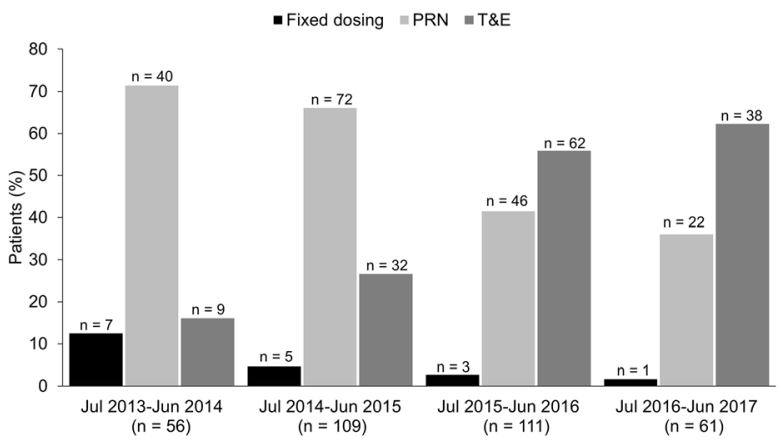

Fig. 3 Proportion of patients treated with intravitreal aflibercept in a fixed, PRN or T\&E regimen between 2013 and 2017. PRN Pro re nata, T\&E treat-and-extend 


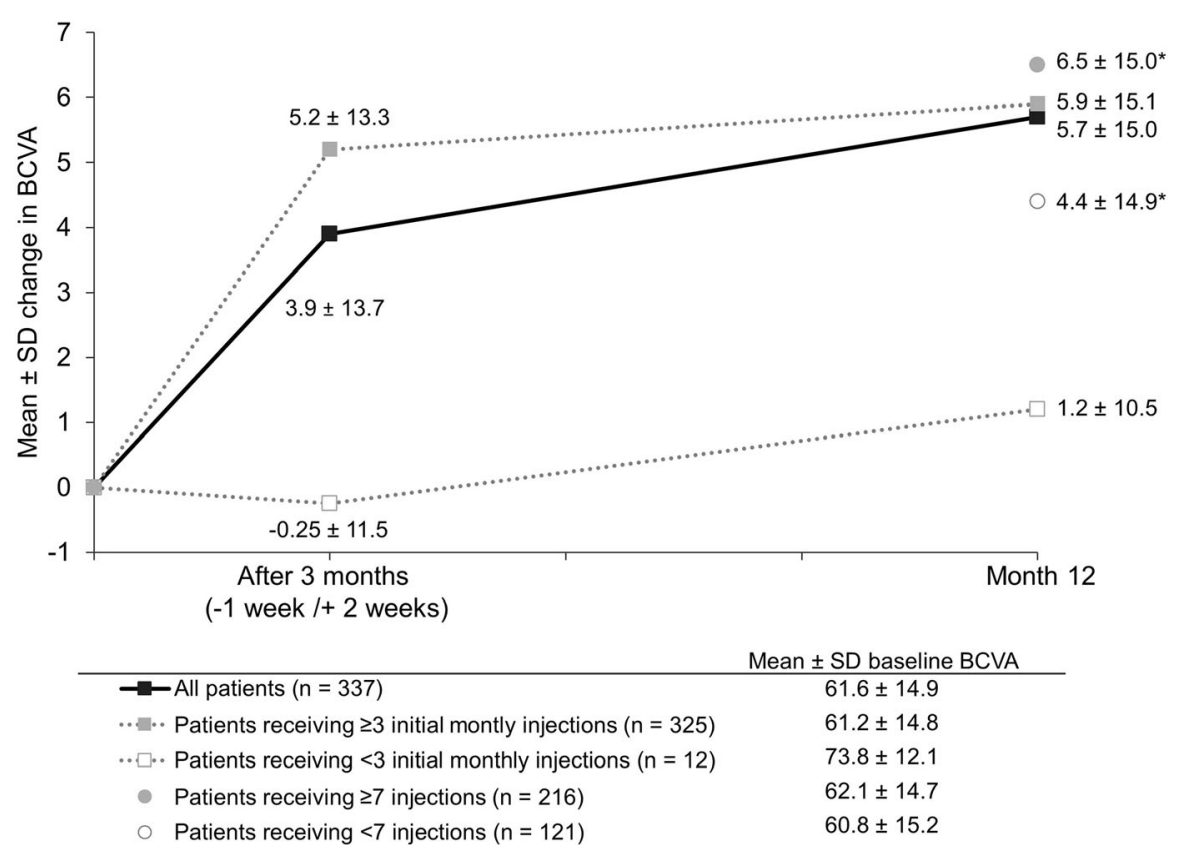

Fig. 4 Mean change in visual acuity by number of injections during the initiation phase and first year of treatment. $B C V A$ Best-corrected visual acuity, $S D$ standard

improvements of $4.4 \pm 14.9$ ETDRS letters (95\% CI 1.7-7.1) in 121 patients who received $<7$ injections. Mean baseline BCVA was also similar to that of the overall population in patients who received $\geq 3$ injections during the first 3 months; however, mean baseline BCVA was numerically higher $(73.8 \pm 12.1$ ETDRS letters; 95\% CI 66.9-80.6) in the 12 patients who received $<3$ injections during the first 3 months. In patients who received $\geq 3$ injections in the first 3 months, mean BCVA improved by $5.2 \pm 13.3$ EDTRA letters (95\% CI 3.8-6.6) after three initial injections and by $5.9 \pm 15.1$ letters $(95 \%$ CI $4.3-7.5)$ after 12 months. This compared with changes of $-0.25 \pm 11.5$ ETDRS letters $(95 \% \mathrm{CI}-6.8$ to 6.3) at the visit following the third injection and $1.2 \pm 10.5$ ETDRS letters (95\% CI -4.7 to 7.1 ) after 12 months in 12 patients who received $<3$ injections in the first 3 months (Fig. 4).

\section{Safety}

In STELLAR, although AEs were listed as the reason for discontinuations in 10 patients, there deviation. Asterisk indicates that 12 -month data were only available for patients receiving $\geq 7$ or $<7$ injections

were no specific AEs reported from patient records by investigators.

\section{DISCUSSION}

To our knowledge, and based on the medical literature, STELLAR provides the first retrospective observational data describing the use of IVT-AFL in clinical practice in Belgium. During the 4-year observation period (2013-2017), there was a shift in treatment regimens from reactive (i.e. PRN) to proactive treatment using a T\&E regimen. More than half (64\%) of patients received $\geq 7$ injections during the first year of treatment. Overall, treatment with IVTAFL was effective, with patients gaining an average of 3.9 ETDRS letters after the first 3 months and 5.7 ETDRS letters after 1 year of IVT-AFL treatment. Although definitive conclusions cannot be drawn since groupings occurred post-baseline, we did observe a greater improvement in visual acuity in patients who received $\geq 7$ injections in the first year of IVTAFL treatment and those who received three 
injections during the first 3 months of IVT-AFL compared with patients who did not.

Despite IVT-AFL being launched in Belgium with a fixed-dose regimen for the first 12 months of treatment (three monthly injections followed by an injection every 8 weeks), IVT-AFL was administered in this manner in only $5 \%$ of patients on average over the 4 -year observation period. In fact, in the first year of observation (2013-2014), IVT-AFL was most commonly administered PRN. The use of the PRN strategy is likely due to the previous experience of investigators with ranibizumab and may account for the mean number of treatment visits being higher than the mean number of injections, as PRN treatment often requires more monitoring visits than injections. The reasons for patient visits (i.e. treatment, monitoring, other) were, however, not collected during the study.

The T\&E protocol is now widely used and can minimize the financial costs and patient burden associated with the need for frequent treatment [18]. Using a T\&E regimen, patients with nAMD can maintain stable visual function over 4 years in real-world clinical practice [19]. In STELLAR, by 2015-2016, the T\&E strategy was the most commonly used approach for a patient's first year of treatment, but it was not until 2018 that IVT-AFL formally received the product licence extension endorsing the T\&E treatment protocol within the first year after three initial monthly doses and an injection 8 weeks later at week 16 .

The proportion of patients who received $\geq 7$ injections during the first 12 months of treatment in STELLAR in Belgium (64\%) was higher than that observed in the French RAINBOW study of treatment-naïve patients and German PERSEUS study of treatment-naïve and previously treated patients, in which 46 and $32 \%$ of patients, respectively, received $\geq 7$ injections $[14,16]$. When making this overall comparison, we note that PERSEUS and RAINBOW, in which patients initiated treatment in 2013-2015 and 2014-2015, respectively, were conducted earlier than STELLAR. However, data from comparative years of STELLAR also indicate that a larger proportion of patients received $\geq 7$ injections in Belgium (48\% in 2013-2014 and 60\% in
2014-2015). The observation of patients receiving 8-10 or more injections in the first year is also consistent with other observational studies [14, 16]. We also noted that only 12 patients $(3 \%)$ who completed 12 months of treatment in STELLAR had not received three monthly injections of IVT-AFL, compared to approximately $20 \%$ of patients who did not receive the initial three monthly doses in RAINBOW and PERSEUS $[14,16]$. Further investigation of these patients found that the reasons for not completing three injections within the first 3 months were varied and included (1) organizational and logistical reasons, which resulted in longer intervals between first three injections and thus the patient not receiving the three injections in a 3-month period; (2) the disease being considered inactive by the treating physician, resulting in follow-up on a reactive PRN basis; and (3) a consequence of comorbidities, such as a delay in injections because of intervening cataract surgery. It was interesting to observe that this small subgroup of patients had a numerically higher BCVA than other patients in the STELLAR study; however, the small number of patients limits the certainty of conclusions that can be drawn regarding BCVA changes in this subgroup.

Although patients were enrolled prospectively in RAINBOW and PERSEUS, STELLAR was a retrospective review in which patients had to have completed 12 months of treatment to be included in the analysis. This study design introduced a selection bias, as patients treated for shorter periods for various reasons were not analysed. In addition, the discontinuation rate after 12 months of treatment (18\%) was lower than expected (25\%), which could suggest that the investigators' selection of patients for enrolment was biased toward those patients who completed the first 12 months.

It is generally accepted that, in regular clinical practice, patients with nAMD receive fewer anti-VEGF injections and have smaller gains in visual acuity than do patients in randomized controlled trials. Consistent with this, patients enrolled in the VIEW studies had larger increases in BCVA from a lower baseline level compared with those in STELLAR [8]. Comparing data across other observational studies, the 
mean baseline visual acuity in the RAINBOW and PERSEUS studies (53.4-56.7 letters) was lower than in the STELLAR study. This provides greater opportunity for improvements from baseline to be demonstrated; however, the mean visual acuity letter gain at 12 months in STELLAR (+ 5.7 letters) was comparable to that reported in those studies ( +5.3 to 5.5 letters) $[14,16]$. Similarly, in a retrospective observational study of IVT-AFL in the UK, at 12 months, mean BCVA improved by +5.0 letters from a mean baseline of 56.3 letters [12]. It is known that older age, better baseline BCVA and larger choroidal neovascularization lesion size are independently associated with lower mean BCVA gains after anti-VEGF therapy $[20,21]$. However, aside from baseline visual acuity, no other baseline disease characteristics or patient demographics were collected in STELLAR. It is therefore unknown whether such differences in patient populations account for differences between STELLAR and other studies.

Despite the limitations described, one particular strength of our study is that data were collected from eight centres, which ensured geographical representativeness of findings and allowed enrolment of a robust number of patients. Although it is difficult to draw overall conclusions encompassing the entire Belgian population of patients with nAMD treated with IVT-AFL, STELLAR provides valuable information about IVT-AFL usage patterns and functional outcomes in clinical practice.

\section{CONCLUSIONS}

Overall, most patients were treated with $\geq 7$ injections of IVT-AFL within the first year of treatment and with $\geq 3$ injections in the first 3 months. The improvement in visual acuity was greater after 3 and 12 months in patients who received this dosing strategy compared with patients who did not receive three injections within the first 3 months and/or $<7$ injections within their first year of treatment. The results of STELLAR showed that functional outcomes achieved in real-world clinical practice in Belgium were consistent with those from other real-world studies of IVT-AFL in treatment-naive patients with nAMD.

The fact that $53 \%$ of the patients were treated with a PRN dosing regimen explains why the number of visits (9.8) was higher than the mean number of injections (7.1) in the first 12 months of treatment. However, there was a shift in the treatment pattern in the 4 years analysed from reactive to proactive treatment.

\section{ACKNOWLEDGEMENTS}

The authors thank the study site investigators and staff and the patients involved in the STELLAR study.

\section{Declarations}

Funding This study was sponsored by Bayer SA/NV. Bayer SA/NV also funded the journal's Rapid Service Fees.

Medical Writing and/or Editorial Assistance Medical writing support was provided by Sarah Feeny (ApotheCom, London, UK) and was funded by Bayer Consumer Care AG, Pharmaceuticals, Switzerland.

Authorship All named authors meet the International Committee of Medical Journal Editors (ICMJE) criteria for authorship for this article, take responsibility for the integrity of the work as a whole, and have given their approval for this version to be published.

Disclosures Joke Ruys: Novartis, Bayer (consultant); Werner Dirven: Novartis, Bayer, Allergan (consultant); Els Mangelschots: Bayer (consultant); Julie Jacob: Novartis, Bayer, Zeiss (consultant), Thea Pharma (research grant); Filip Mergaerts: Novartis, Bayer (symposia); Alexandra Kozyreff: Bayer, Novartis, Allergan, Abbvie, Mylan (consultant).

Compliance with Ethics Guidelines Approvals from independent ethics committees were obtained for all participating centres prior to study start (Electronic Supplementary Material Table). 
Data Availability Availability of the data underlying this publication will be determined according to Bayer's commitment to the EFPIA/ PhRMA "Principles for responsible clinical trial data sharing". This pertains to scope, time point and process of data access. As such, Bayer commits to sharing upon request from qualified scientific and medical researchers patients-level clinical trial data, study-level clinical trial data, and protocols from clinical trials in patients for medicines and indications approved in the USA and EU as necessary for conducting legitimate research. This applies to data on new medicines and indications that have been approved by the EU and US regulatory agencies on or after 1 January 2014.Interested researchers can use www.clinicalstudydatarequest.com to request access to anonymized patient-level data and supporting documents from clinical studies to conduct further research that can help advance medical science or improve patient care. Information on the Bayer criteria for listing studies and other relevant information is provided in the study sponsors section of the portal. Data access will be granted to anonymized patientlevel data, protocols and clinical study reports after approval by an independent scientific review panel. Bayer is not involved in the decisions made by the independent review panel. Bayer will take all necessary measures to ensure that patient privacy is safeguarded. In this study, data were collected in an aggregated manner per centre.

Open Access. This article is licensed under a Creative Commons Attribution-NonCommercial 4.0 International License, which permits any non-commercial use, sharing, adaptation, distribution and reproduction in any medium or format, as long as you give appropriate credit to the original author(s) and the source, provide a link to the Creative Commons licence, and indicate if changes were made. The images or other third party material in this article are included in the article's Creative Commons licence, unless indicated otherwise in a credit line to the material. If material is not included in the article's Creative Commons licence and your intended use is not permitted by statutory regulation or exceeds the permitted use, you will need to obtain permission directly from the copyright holder. To view a copy of this licence, visit http://creativecommons.org/licenses/by$\mathrm{nc} / 4.0 /$.

\section{REFERENCES}

1. McCannel CA (ed). 2019-2020 Basic and clinical science course. San Francisco: American Academy Of Ophthalmology; 2019.

2. Chen Y, Bedell M, Zhang K. Age-related macular degeneration: genetic and environmental factors of disease. Mol Interv. 2010;10(5):271-81.

3. Schmidt-Erfurth U, Chong V, Loewenstein A, et al. Guidelines for the management of neovascular agerelated macular degeneration by the European Society of Retina Specialists (EURETINA). Br J Ophthalmol. 2014;98(9):1144-67.

4. American Academy of Ophthalmology. Preferred practice. Pattern ${ }^{\circledR}$ Guidelines age-related macular degeneration. San Francisco: American Academy of Ophthalmology; 2015.

5. Bakri SJ, Thorne JE, Ho AC, et al. Safety and efficacy of anti-vascular endothelial growth factor therapies for neovascular age-related macular degeneration: a report by the American Academy of Ophthalmology. Ophthalmology. 2019;126(1):55-63.

6. Busbee BG, Ho AC, Brown DM, et al. Twelve-month efficacy and safety of $0.5 \mathrm{mg}$ or $2.0 \mathrm{mg}$ ranibizumab in patients with subfoveal neovascular age-related macular degeneration. Ophthalmology. 2013;120(5):1046-56.

7. Martin DF, Maguire MG, Fine SL, et al. Ranibizumab and bevacizumab for treatment of neovascular agerelated macular degeneration: 2-year results. Ophthalmology. 2012;119(7):1388-98.

8. Heier JS, Brown DM, Chong V, et al. Intravitreal aflibercept (VEGF trap-eye) in wet age-related macular degeneration. Ophthalmology. 2012;119(12): 2537-48.

9. Schmidt-Erfurth U, Kaiser PK, Korobelnik JF, et al. Intravitreal aflibercept injection for neovascular age-related macular degeneration: 96-week results of the VIEW studies. Ophthalmology. 2014;121(1): 193-201.

10. Bayer Pharma AG. Eylea ${ }^{\circledR}$ (aflibercept solution for injection) summary of product characteristics. Leverkusen: Bayer Pharma AG; 2020. 
11. Ohji M, Takahashi K, Okada AA, et al. Efficacy and safety of intravitreal aflibercept treat-and-extend regimens in exudative age-related macular degeneration: 52- and 96-week findings from ALTAIR. Adv Ther. 2020;37(3):1173-87.

12. Almuhtaseb H, Johnston RL, Talks JS, Lotery AJ. Second-year visual acuity outcomes of nAMD patients treated with aflibercept: data analysis from the UK Aflibercept Users Group. Eye (Lond). 2017;31(11):1582-8.

13. Almuhtaseb H, Kanavati S, Rufai SR, Lotery AJ. Oneyear real-world outcomes in patients receiving fixed-dosing aflibercept for neovascular age-related macular degeneration. Eye (Lond). 2017;31(6): 878-83.

14. Framme C, Eter N, Hamacher T, et al. Aflibercept for patients with neovascular age-related macular degeneration in routine clinical practice in Germany: 12 month outcomes of PERSEUS. Ophthalmol Retina. 2018;2(6):539-49.

15. Ozturk M, Harris ML, Nguyen V, Barthelmes D, Gillies MC, Mehta H. Real-world visual outcomes in patients with neovascular age-related macular degeneration receiving aflibercept at fixed intervals as per UK licence. Clin Exp Ophthalmol. 2018;46(4):407-11.

16. Weber M, Velasque L, Coscas F, Faure C, Aubry I, Cohen SY. Effectiveness and safety of intravitreal aflibercept in patients with wet age-related macular degeneration treated in routine clinical practices across France: 12-month outcomes of the RAINBOW study. BMJ Open Ophthalmol. 2019;4(1): e000109.

17. European Medicines Agency. Guideline on good pharmacovigilance practices (GVP). Amsterdam: European Medicines Agency; 2017.

18. Ross AH, Downey L, Devonport H, et al. Recommendations by a UK expert panel on an aflibercept treat-and-extend pathway for the treatment of neovascular age-related macular degeneration. Eye. 2020:1-10. https://doi.org/10.1038/s41433-0190747-x.

19. Traine PG, Pfister IB, Zandi S, Spindler J, Garweg JG. Long-term outcome of intravitreal aflibercept treatment for neovascular age-related macular degeneration using a "treat-and-extend" regimen. Ophthalmol Retina. 2019;3(5):393-9.

20. Lanzetta P, Cruess AF, Cohen SY, et al. Predictors of visual outcomes in patients with neovascular agerelated macular degeneration treated with antivascular endothelial growth factor therapy: post hoc analysis of the VIEW studies. Acta Ophthalmol. 2018;96(8):e911-e918918.

21. Ho AC, Saroj N, Baker K, et al. Impact of baseline characteristics on treatment response to intravitreal aflibercept injection for wet age-related macular degeneration. Ophthalmol Retina. 2018;2(7): 676-83. 